\title{
Lagrange as a Historian of Mechanics
}

\author{
Agamenon R. E. Oliveira \\ Polytechnic School of Rio de Janeiro, Federal University of Rio de Janeiro, Rio de Janeiro, Brazil \\ Email: agamenon.oliveira@globo.com
}

Received August $5^{\text {th }}$, 2013; revised September $6^{\text {th }}, 2013$; accepted September $15^{\text {th }}, 2013$

\begin{abstract}
Copyright (c) 2013 Agamenon R. E. Oliveira. This is an open access article distributed under the Creative Commons Attribution License, which permits unrestricted use, distribution, and reproduction in any medium, provided the original work is properly cited.
\end{abstract}

\begin{abstract}
In the first and second parts of his masterpiece, Analytical Mechanics, dedicated to static and dynamics respectively, Lagrange (1736-1813) describes in detail the development of both branches of mechanics from a historical point of view. In this paper this important contribution of Lagrange (Lagrange, 1989) to the history of mechanics is presented and discussed in tribute to the bicentennial year of his death.
\end{abstract}

Keywords: History of Mechanics; Epistemology of Physics; Analytical Mechanics

\section{Introduction}

Lagrange was one of the founders of variational calculus, in which the Euler-Lagrange equations were derived by him. He also developed the method of Lagrange multipliers which is a manner of finding local maxima and minima of a function subjected to constraints. He developed the method for solving differential equations known as the parameter variation method. In addition, he applied differential calculus to the theory of probabilities and did notable work in obtaining the solution of algebraic equations. Furthermore, in calculus Lagrange introduced a new approach for the interpolation of the Taylor (1685-1731) series. His famous treatise known as the Theory of Analytical Functions contains the path that leads to the foundation of group theory, anticipating the work of Evariste Galois (18111832).

In mechanics Lagrange studied specific problems, such as the three-body problem related to motion of the earth, sun and moon. By means of his Analytical Mechanics, he transformed Newtonian mechanics (Newton, 1952) into a branch of analysis, Lagrangian mechanics, which was a result of the application of the variational calculus to mechanical principles. Through this work, rational mechanics was able to fulfill the long desired Cartesian aim of becoming a branch of pure mathematics.

In relation to problems later called applied mechanics, Lagrange, in the works known as the Mélanges de Turin, studied the propagation of sound, making an important contribution to the theory of vibrating strings. He used a discrete mass model to represent string motion consisting of $\mathrm{n}$ masses joined by weightless strings. Then he solved the system of $n+1$ differential equations, when $\mathrm{n}$ tends to infinity to obtain the same functional solution proposed by Euler (1707-1783). Lagrange also studied the integration of differential equations and made various applications to topics such as fluid mechanics, where he introduced the Lagrangian function

Lagrange's Analytical Mechanics was published in 1788, crowning a series of works and other important contributions previously developed by d'Alembert (1717-1783) and Euler (Euler, 1952). This book presents a model of formalized theory in the same meaning that is now understood by modern physicists. The logical unity of this theory is based on the least action principle. However, the two dimensions of formalization and unification are the main characteristics of Lagrange's method.

\section{Lagrange: A Biographical Note}

Joseph-Louis Lagrange was born in Turin on January 25, 1736, under the name of Giuseppe Lodovico Lagrangia (Figure 1). His father was Giuseppe Francesco Lodovico Lagrangia and was Treasurer of the Office of Public Works and Fortifications in Turin. His mother was Teresa Grosso, the only daughter of a medical doctor from Cambiano near Turin. Lagrange was the eldest of their 11 children, but one of only two to live to adulthood.

Turin became the capital of the kingdom of Sardinia in 1720, sixteen years before Lagrange's birth. His family had French connections on his father's side. His grandfather was a French

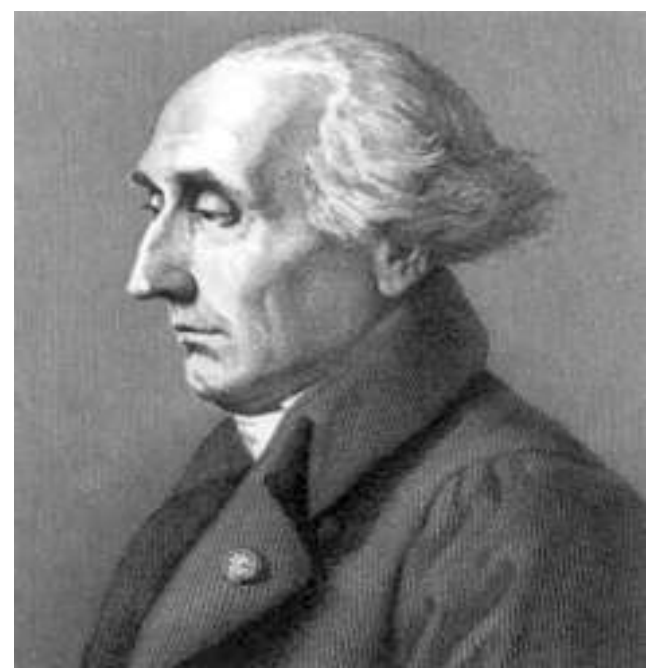

Figure 1.

Joseph-Louis Lagrange (1736-1813). 
cavalry captain who had left France to work for the Duke of Savoy. For this reason, Lagrange always leant towards his French ancestry. When he was young he signed his name Lodovico LaGrange or Luigi Lagrange, using the French form of his family name.

Lagrange's interest in mathematics began when he read a copy of Halley's 1693 work on the use of algebra in optics. He was also attracted to physics by the excellent teaching of Francesco Ludovico Beccaria (1716-1781) at the College of Turin, leading him to decide to follow a career in mathematics.

Returning to Mécanique Analytique, it was written by Lagrange during his period in Berlin and was approved for publiccation by a committee from the Academy of Sciences consisting of Laplace (1749-1827), Cousin, Legendre (1752-1833) and Condorcet (1743-1794). This book summarized all the work done in the field of mechanics since the time of Newton (1642-1727), being notable for its use of the theory of differenttial equations. In 1810, he commenced a thorough revision of his masterpiece, but he was able to complete only about twothirds of this before his death in Paris, on April 10, 1813. He was buried in the same year in Panthéon in Paris. The French inscription on his tomb reads:

Joseph-Louis Lagrange. Senator. Count of the Empire. Grand Officer of the Legion of Honour. Grand Cross of the Imperial Order of the Reunion. Member of the Institute and the Bureau of Longitude. Born in Turin on 25 January. Died in Paris on 10 April 1813.

\section{Science and the French Revolution}

The French revolution has a great importance as a fundamental transformation of European society from the social, political and scientific viewpoints. Besides the intellectual and cultural changes before the takeover of power by the bourgeoisie in France with direct consequences to the scientific production over a long period of his history, we should also mention other factors and aspects of this context.

The first important thing to note is the need of the new regime for new institutions in order to criticize and fight against the ideas of the ancien regime. These new institutions also appeared within the educational system as the best way to change mentalities and to prepare new technical and political elite to give continuity to the project of a new society announced by the Revolution.

The transformations in the educational system of France implied significant modifications in technical and professional education, because new creeds, new knowledges and new technologies had progressed, making it necessary to teach them. The development of engineering and its teaching is important in this context. A reformation of engineering instruction was necessary also because war with other European countries had stimulated the construction of fortifications, roads and bridges, and the development of artillery. This new context propelled France to apply scientific principles to industry, with the result that the new engineering had to provide universal scientific knowledge as well as tools and methods applicable in a diverse range of practical situations (Belhoste, 2003). As we know, Lagrange played an important role in the context of these transformations. He was the first professor of analysis, appointed for the opening of the École Polytechnique in 1794. In 1795 the École Normale was founded with the aim of training school teachers. Lagrange taught courses on elementary ma- thematics there.

\section{Historical Considerations in Analytical Mechanics}

\section{First Part: Statics}

Lagrange began his history of statics by defining this discipline associated with the concept of force. He states:

Statics is the science of forces in equilibrium. We think, in general, of force or power as a cause, anything that impresses or tend to impress motion on the body under consideration; it is also by the quantity of impressed motion, or by its tendency, that a force or power must be estimated.

According to him, the objective of statics is to provide the laws that govern equilibrium. In this sense equilibrium appears as the destruction of several forces that oppose and annihilate them. These laws are based on three general principles, namely: a) the equilibrium of the lever; b) the composition of motions; c) virtual velocities. It is, thus, in the context of the historical development of these three principles that Lagrange rebuilds the history of statics.

Lagrange considers Archimedes (287 - 212 b. C.) as the only scholar from ancient times who had produced a theory of Mechanics, which is contained the latter's two books named Aequiponderantibus. Archimedes was also the author of the principle of the lever (Dijksterhuis, 1987). In modern times the contributions of Stevin (1548-1620), in his Statics, and Galileo (1564-1642), in his Dialogues (Discorsi) about motion, had transformed Archimedes' demonstration into a much more simple and useful concept (Galileo, 1988). However, it seemed to Lagrange that ancient mathematicians did not know of a method to generalize the principle of the lever to other simple machines, notably the inclined plane. This problem is also posed to the first modern mathematicians. Stevin presented the first exact solution to this problem independent of the lever theory. These considerations led him to the impossibility of perpetual motion. (See Elements of Statics and Hypomnemata Mathematica)

The second fundamental principle of equilibrium is the composition of motions. It is assumed that when two forces are acting in different directions on a body, these two forces are equivalent to one following the diagonal of the parallelogram. In all cases when there are several forces, the composition of two forces leads to a single force representing the whole system. For the equilibrium condition this force must be zero if there is no fixed point. This conclusion is found in any book of Statics, particularly in Varignon's new mechanics (Blay, 1992). In addition he derived a theory of machines using this principle alone.

The origin of this principle is attributed by Lagrange to Galileo, specifically in the second Proposition of the Fourth Journey in his Dialogues (Discorsi). Lagrange remarks that Galileo does not consider the entire importance of the Principle in his theory of equilibrium.

The theory of composed motions can also be found in the writings of Descartes (1596-1650), Roberval (1602-1675), Mersenne (1588-1648), and Wallis (1616-1703). As mentioned above, Varignon (1654-1722) used this principle for machines in equilibrium. His project of a new mechanics, presented in 
1687, had this objective.

Let us look at the third principle, virtual velocities. This is understood as the velocity acquired by a body whose equilibrium is not maintained. The principle states that for the equilibrium the powers are in inverse ratio to the virtual velocities, estimated in the direction of the powers. Lagrange attributes the discovering of this principle to Galileo in his Dialogues (Discorsi). (See the Scholium of the second proposition of the third Dialogue) In this context, Galileo also defines the moment of some weight or of some power applied to a given machine as an action, energy, or impetus to move the machine in a way that equilibrium is maintained between two powers with the condition that the moments are equals and in contrary sense. The moment is always proportional to a power (force) multiplied by its virtual velocity.

This notion of moment that came from Galileo was adopted by Wallis in his Mechanics, published in 1669. He emphasizes the principle of equality of moments as the main foundation for Statics, and thus applies this theory to machines. In parallel, Descartes summarizes Statics in a unique principle, which in fact is the same as proposed by Galileo, though presented in a new and general form. This principle is based on the force necessary to elevate a weight to a height. Afterwards it was used extensively to evaluate the capacity of a given machine or to compare machines with different capacities. The birth of applied mechanics, mainly with Lazare Carnot (1753-1823), uses this mechanical model extensively (Oliveira, 2012).

Another important principle described by Lagrange is Torricelli's principle. He was a famous disciple of Galileo and his principle is directly related to Galileo's concepts, or even are a consequence of Galileo's analysis. The principle states that when a system of bodies is in equilibrium its center of gravity is in the lowest position. In the condition of equilibrium the center gravity cannot go up or down due to infinitely small variations of position.

Lagrange enunciated the principle of virtual velocities in a general form as follows:

If in any system of bodies or material points, any one of them is submitted to forces, but the system is in the position of equilibrium and therefore we apply any small motion, as a consequence each point describes an infinitely small space which will express its virtual velocity; the addition of all forces multiplied by the displacement of its points of application following the direction of the force will be always zero, since we adopt as positive the displacements in the direction of the forces and as negative the displacements in opposite sense to the forces.

Lagrange also remarks that was Jean Bernoulli (1667-1748) the first to realize the great generality of the principle of virtual velocities, as well as its usefulness to solve statics problems. He mentions the letter addressed by Jean Bernoulli to Varignon in 1717 about this principle and other important developments, such as that of Maupertuis (1698-1759), who in 1740 proposed to Paris Academy of Sciences the name of the Law of Rest, and Euler who developed in his Memorials to the Berlin Academy in 1754.

\section{Second Part: Dynamics}

As in the previous section, Lagrange begins this topic defining dynamics by the effect that forces can cause on bodies, by accelerating or decelerating them. In addition, this science was entirely developed by modern mathematicians and physicists. Again, the name of Galileo arises as the one who presented the first fundamental concepts of dynamics. In addition, Galileo developed the kinematics of the free fall of heavy bodies, in which the law of inertia is also constantly present in the free fall, but also the motion of projectiles. Before Galileo forces were only discussed in the context of equilibrium conditions. In spite of the simplicity involving the falling of heavy bodies and the motion of projectiles, the determination of the laws governing these phenomena were unknown until Galileo. He took the first step and opened the way to advancing mechanics. Lagrange then refers to Galileo's master piece, calling it the Dialogues About the New Science, published in Leiden in 1637. Obviously, he means the Discorsi.

Following the development of mechanics, Lagrange studied Huygens' contributions, especially the latter's findings on pendulum motion and the mathematization of centrifugal force, which were fundamental steps towards the discovery of universal gravitation. Huygens' construction of a bridge between Galileo and Newton was of great importance (Taton, 1982).

Mechanics became a new science due to Newton's book known as Mathematical Principles, which appeared for the first time in 1687. With the invention of infinitesimal calculus it was possible to transform the laws of motion into analytical equations.

The theory of motion produced by driven forces is based on general laws of any motion impressed on a given body. These laws are derived from known principles which are inertia force and composed motion. As in Statics, Lagrange looks for general principles governing dynamics phenomena using the category of force as a unifying concept. Thus, he identifies these two already mentioned principles as the most general and fundamental. It is in the context of these two principles where Lagrange develops his historical considerations.

Galileo realized that the first principle enunciated and derived from the laws governing the motion of projectiles through the composition of horizontal motion with constant velocity with the vertical up and down motion modified by gravity acceleration. The case of falling bodies with the velocity acquired being proportional to the elapsed time, or the vertical displacement proportional to the time squared, was an important achievement made by Galileo using geometrical considerations in addition to experimental measurements with inclined planes.

After Galileo, Huygens (1629-1695) discovered the laws of centrifugal forces of bodies in circular motion with constant velocity, and used this knowledge to compare forces. As a result weight on the surface of the earth could be calculated as a centrifugal force. This was done in his Horologium Oscilatorum, published in 1673 (Huygens, 1673).

Newton generalized this theory to any kind of curve, thereby developing the science for varied motions with accelerated forces. He used a geometrical method and occasionally analytical calculation, though instead of differential methods he applied the series method. After Newton the majority of mathematicians that developed the theory of motion only generalized Newton's theorems, introducing differential expressions to solve many kinds of problems.

Lagrange then explains how to solve a dynamical problem by using three different perpendicular directions and decomposing forces and accelerations in these directions. The forces in any direction can be calculated by equating on one side forces and 
on the other the second the differential of space divided by the first differential of time squared. For curved trajectories, the decomposition had to be done in normal and tangential directions. Lagrange does not mention that it was Euler who applied this for the first time in 1752 in a manner different to Newton's second law (Truesdell, 1983).

Lagrange describes how the problem of shocks between hard bodies was studied, explaining the result of these interactions by means of the analysis of quantities of motion. He mentions that it was Descartes who first realized the principle behind this phenomenon. However, as confirmed by Lagrange, Descartes made a mistake in the application of the principle because he considered that the absolute quantity of motion was always conserved. After Descartes, Wallis was the first to have a clear idea of the principle and used it to discover the laws for the communication of motion in the context of shocks between hard and elastic bodies, as presented in his Philosophical Transactions, published in 1669, as well as in the third part of his treatise De Motu, which appeared in 1671.

One of the most important passages of Lagrange's text is dedicated to the d'Alembert principle. The Treatise of Dynamics written by d'Alembert and published in 1743 presented a general and direct method to solve, or at least to obtain the equations for, practically any dynamic problem (d'Alembert, 1743). The method proposed transformed the laws of bodies in motion to its equilibrium, thereby relating dynamics to statics. The principle enunciated by d'Alembert generalizes the work of some previous mathematicians, such as Jacques Bernoulli (16541705), with great simplicity.

We can enunciate the principle by studying the motion of various bodies which tend to move with velocities and in a direction so that changes in both are caused by their interactions. It is possible to visualize these motions as composed by what was really acquired and others that are destroyed in the interactions. If we consider only the final motions the bodies animated with them are in equilibrium.

It is important to emphasize that d'Alembert made useful applications to mechanical problems. However, this principle does not provide the necessary equations to solve different dynamical problems but rather provides the means to derive the equations from the conditions of equilibrium. Thus, by combining the principle with known principles of equilibrium, such as the lever principle or that of the composition of forces, we can find the equations for each problem with the help of some more or less complicated constructions. The difficulty is to evaluate the forces destroyed.

As discussed previously, the principle of virtual velocities leads us to a very simple analytical method to solve static problems. This same principle combined with the d'Alembert principle also provides a similar method to solve dynamical problems. Explaining this approach in more detail, the application of the principle of virtual velocities consists of the following methodology. For a given system containing several bodies that can be reduced to points being acted upon by any kind of force, if we apply to the system a small motion, each body displaces an infinitesimal space. If we multiply each force by the displacement of its point of application and add them for the whole system, the result is zero.

If we suppose the system is in motion, and considering that the velocities of each body can be decomposed in three fixed and perpendicular directions, the decreasing of these velocities will represent the motions lost along the same directions and their increasing will be the motions lost in the opposite directions. Thus, these lost motions will be expressed in general by the mass multiplied by the element of velocity and divided by the time element, and they will have contrary directions to the velocities. Using this approach it is possible to obtain a general formula to represent the motions of bodies which will provide a solution for any dynamic problem.

One of the advantages of the above mentioned formula is that it immediately offers the general equations which encompass the principles and known theorems about the conservation of living forces, the conservation of the motion of the center of gravity, the conservation of the moments of rotation motion, or the principle of areas and the principle of least action. These principles can be considered the general achievements of the dynamic laws and are the primary principles of this science. With this statement Lagrange proposed to explain its origins and developments.

The first mentioned principle, the conservation of living forces, was first presented by Huygens, but in a different form to how it is now known. In its origins, the principle represented the equality between the descending and raising of the center of gravity of several heavy bodies, in which descending in a group but raising separately, using the known properties of the gravity center, the space displaced by this center in any direction is expressed by adding the products of the mass of each body by the space displaced in the same direction divided the total mass. On the other hand, using Galileo's theorems, the vertical displacement of a heavy body is proportional to the square of the velocity acquired in free descent, as well as what can be reached by raising it to the same height. Based on these considerations, Huygen's principle can consider the motion of heavy bodies in which the sum of the products of the masses by the square of the velocities at each time is the same, since that the bodies motion be conjunctly in any way, or that they displaces freely to the same vertical heights. Huygens made these remarks in a short paper on the methods used by Jacques Bernoulli and the Marquis l'Hopital (1661-1704). Obviously, the principle postulated by Huygens is a particular application of the more general principle of conservation of energy, a concept which would appear only in the middle of the nineteenth century.

After these achievements, Daniel Bernoulli (1700-1782) derived from this principle the laws of fluid motion in vessels, which had not been previously dealt with. He reached this general principle in the Berlin memorials, published in 1748 (Bernoulli, 1968).

The great advantage of this principle is that it easily provides an equation between the velocities of the bodies and the variables which calculate their position in space in such a manner that, due to the characteristics of the problem, all these variables are reduced to one, with this equation being sufficient to solve completely the problem.

The second principle is due to Newton who, at the beginning of his Principia, demonstrated that the state of rest or motion of the center of gravity of several bodies does not change through their reciprocal action. This implies that the center of gravity of the system is at rest or in uniform linear motion unless it meets some exterior obstacle. Obviously this principle is useful to determine the center of gravity motion independently of individual bodies' motions, as it can provide three equations between the bodies' coordinates and time.

The third principle, more recent than the other two, seems to 
have been discovered simultaneously in different ways by Euler, Daniel Bernoulli, and Le Chevalier d'Arcy (1723-1779). According to Euler and Daniel Bernoulli, this principle involves considering the motion of several bodies around a fixed center. Hence, the sum of the products of the mass of each body by the circular velocity around the center is always independent of the mutual action among the bodies and is conserved unless some exterior obstacle is found.

The principle enunciated by d'Arcy, which appeared in the Memorial he presented to the Paris Academy of Sciences in 1746 , is that the sum of the products of the mass of each body by the area described by its vector radius around the fixed center is always proportional to time. This principle generalizes Newton's theorem about areas due to any centripetal forces.

Finally, the fourth principle called the least action, in an analogy with Maupertuis' principle with the same name which had become famous. It involved considering the motion of several bodies acting among them and then taking the sum of the products of the masses by the velocities and the spaces described is a minimum. Maupertuis had derived this from the laws of the reflection of light and refraction, as well as of mechanical shocks. These studies appear in two Memorials, one presented to the Academy of Sciences of Paris in 1744 and the other to the Berlin Academy (Maupertuis, 1744).

Before being completely established as a principle, Euler made the first approach to it in his treatise on isoperimetric curves, printed in Lausanne in 1744, postulating that in the trajectories described by central forces, the integral of the velocity multiplied by the curve element is always a maximum or a minimum. This property which Euler did not recognize except for isolated bodies, as he mentioned, was extended to any motion of bodies acting among themselves, leading to this new general principle in which the sum of the products of the masses by the integrals of the velocities multiplied by the space elements is constant and a maximum or a minimum. It is a simple consequence of mechanical laws. This principle combined with the principle of the conservation of living forces following the rules of variational calculus directly provides all the necessary equations to solve each problem giving rise to a method to solve problems of motion.

\section{Final Remarks and Conclusion}

One of the aims most sought by physicists along the years has been the finding of a principle, the simplest possible, or some basic fundamental principles, which could fit all natural phenomena. Some tried to do this, as Lagrange's analysis demonstrates. D'Alembert did the same. In his Preliminary Discourse in the Treatise on Dynamics, one reads: If the principle of the inertia of force, of composed motion, and of equilibrium, are essentially different from each other, as we cannot prohibit happening; and if, on the other hand, these three principles are sufficient for mechanics, one can reduce this science to the least number of principles possible, and assume that on these three principles there can be established all the laws of motion for any body in any circumstances, as I have accomplished in this work.

In his famous Fundamental Principles of Equilibrium and
Motion, published in 1803, Lazare Carnot states: There are two ways to see mechanics and its principles. The first one is by considering it as a theory of forces, the causes that impress motion. The second is by considering it as a theory of motions themselves. Here an important remark has to be made. Lagrangian mechanics is the development of mechanics using the second approach, the analysis of motions by themselves as defined by Carnot. However, with respect to history of mechanics, Lagrange adopts the concept of force to both statics and dynamics to explain its internal development, obviously because of the late development of the other concepts associated with motion that we know nowadays as the methods of energy.

Another important contribution in the historical considerations of mechanics made by Lagrange is that it highlights some developments which are not completely clear in the current literature. One example is his correct interpretation of d'Alembert's principle. As we know, from reading most mechanics or physics textbooks, this principle is always presented as a method to reduce a dynamical problem into a statics one. Lagrange, as in the original version of the d'Alembert principle, only considers the possibility of equilibrium where motions are destroyed. In other words, equilibrium means the conservation of the quantity of motion.

Lastly, the importance attributed by Lagrange to include in his masterpiece historical considerations about the development of mechanics, only confirms that the internal development of science is not independent of its historical development.

\section{REFERENCES}

Belhoste, B. (2003). La formation d'une technocratie. Paris: Belin, rue Féron.

Bernoulli, D. (1968). Hydrodynamics. New York: Dover Publication, Inc.

Blay, M. (1992). La science du mouvement: De galilée à lagrange. Paris: Belin, rue Féron.

D’Alembert, J. L. (1921). Traité de dynamique. Paris: Gauthiers-Villars et Cie Éditions.

Dijksterhuis, E. J. (1987). Archimedes. Princeton, NJ: Princeton University Press.

Euler, L. (1952). Methodus inveniendi lineas curvas maximi minimive proprietates gaudentes. In leonhardi euleri opera omnia, s. I, vol. XXIV, Lausanne.

Galileu, G. (1988). Discurso sobre as duas novas ciências, museu de astronomia e ciências afins, Rio de Janeiro.

Huygens, C. (1673). Horologium oscilatorum. Paris: Albert Blanchard Library.

Lagrange, J. L. (1989). Mécanique analytique. Paris: Éditins Jacques Gabay.

Maupertuis, P. L. M. (1744). Accord des différents lois de la nature qui avaient jusqui'ici paru incompatibles. Memoires de l'Academie des Sciences de Paris.

Newton, I. (1952). Mathematical principles of natural philosophy. London: Great Books of the Western World.

Oliveira, A. R. E. (2012). The role of the concept of work in the development of applied mechanics. Rome: SISFA.

Taton, R. (1982). Huygens et la France. Paris: Librairie Philosophique J. Vrin.

Truesdell, C. (1983). Essays in the history of mechanics. Berlim: SpringerVerlag. 